\title{
COGNITIVE DISORDERS IN PATIENTS WITH PARKINSON'S DISEASE ON THE BACKGROUND OF AUTOIMMUNE PATHOLOGY
}

\author{
Tetiana Cheboraka \\ Department of Neurology No. 1 \\ Shupyk National Medical Academy of Postgraduate Education \\ 9 Dorohozhytska str., Kyiv, Ukraine, 04112 \\ tatyana.neurology@gmail.com \\ Tatyana Slobodin \\ Department of Neurology No. 1 \\ Shupyk National Medical Academy of Postgraduate Education \\ 9 Dorohozhytska str., Kyiv, Ukraine, 04112 \\ Yurii Holovchenko \\ Department of Neurology No. 1 \\ Shupyk National Medical Academy of Postgraduate Education \\ 9 Dorohozhytska str., Kyiv, Ukraine, 04112
}

\begin{abstract}
Parkinson's disease (PD) is a progressive neurodegenerative disease characterized by the predominantly dopaminergic neuronal black matter degeneration. The multicentre study of PRIAMO (PaRkinson \& non Motor symptom) showed that in $98.6 \%$ of cases, non-motor symptoms (NMS) were observed in patients with PD. Cognitive impairment is one of the most common NMS PD. According to these studies, cognitive dysfunction develops in most patients at an early stage of the disease, with mild to moderate cognitive impairment. In the later stages of the disease, dementia occurs in $80 \%$ of patients with PD.

Aim of the research. To study the peculiarities of cognitive impairment in patients with PD and autoimmune thyroiditis (AIT), their association with motor and non-motor disorders, and to assess the impact on the quality of life of patients.

Materials and methods of the research. 109 patients with PD aged 47 to 75 years were examined. The main group consisted of patients with IA and IB subgroups, control - IIA and IIB subgroups. General clinical and neurological examinations, evaluation of motor functions by the Unified Parkinson's disease Rating Scale (UPDRS), neuropsychological testing (MMSE, MoCA, FAB, BDI), Hamilton's Alert Scale (HARS), scale used for assessing autonomic disorders in patients with PD and Parkinson's disease questionnaire (PDQ-39), statistical analysis using the "Statistica 6.0" program.

Results. Neuropsychological testing showed that MMSE, MoCA, and FAB obtained from patients with PD and AIT are lower compared to patients with PD. Analyzing the indicators of MMSE, FAB, and MoCA scales in dynamics, a statistically significant difference was found in subgroups of IB and IIB; in subgroups of IA and IIA - was not observed. During the analysis of the results of the neuropsychological testing, no association was found at the statistically significant level in the subgroups of IA and IB between the duration of the disease and MMSE, MoCA, and FAB scales. In the IA subgroup, in the initial review, feedback was observed on a statistically significant level of average strength between the level of anxiety and the indicator of the MMSE scale, an indicator of the MoCA scale. In the IA subgroup, there was no relationship at the statistically significant level between the indicators of neuropsychological testing (MMSE, MoCA, FAB) and motor and non-motor manifestations (depression, vegetative disorders). There was a connection at a statistically significant level between the indicators of neuropsychological testing (MMSE, MoCA, FAB) and motor and non-motor manifestations of PD in patients who received anti-parkinsonian therapy for a long period of time. The negative influence of the level of cognitive impairments on the quality of life of patients with PD and AIT was revealed, indicating the high medical and social significance of these violations.

Conclusions. Neuropsychological testing showed that MMSE, MoCA, FAB scores in patients with PD and AIT (IB subgroup) were lower compared to patients with PD (IIB subgroup). There was a connection at a statistically significant level between the indicators of neuropsychological testing (MMSE, MoCA, FAB) and motor and non-motor manifestations of PD in patients who received anti-parkinsonian therapy for a long period of time. The negative influence of the level of cognitive impairments on the quality of life of patients with PD and AIT was revealed, indicating the high medical and social significance of these violations.

Keywords: Parkinson's disease, autoimmune thyroiditis, cognitive impairment, depression, anxiety, autonomic disorders, motor disorders, quality of life.
\end{abstract}




\section{Introduction}

Parkinson's disease (PD) is a progressive neurodegenerative disease characterized by the degeneration of predominantly dopaminergic neurons of the black substance [1]. Before the first motor manifestations of PD occur, about $40-60 \%$ of dopaminergic neurons are lost. The period of the disease during which the neurodegenerative process progresses in the absence of classical motor symptoms is called the "prodromal phase of PD" [2]. During the "prodromal phase of PD", a wide range of non-motor symptoms (NMSs) is observed: vegetative, sensory, cognitive, affective, behavioral and psychotic disorders $[3,4]$. The multicentric study of PRIAMO (PaRkinson \& non Motor symptom) showed that in $98.6 \%$ of cases, NMS was observed in patients with PD [5]. Cognitive impairment is one of the most common PDs NMS. [6]. According to these studies, cognitive dysfunction develops in most patients at an early stage of the disease, with mild to moderate cognitive impairment. Patients have neurodynamic disorders in the form of slowness, astonity (bradyphenia), decreased attention, functional capacity and regulatory disturbances that reflect dysfunction of the frontal lobes [7, 8]. In the later stages of the disease, dementia occurs in $80 \%$ of patients with PD $[9,10]$. The development of dementia is associated with a decrease in the effectiveness of antiparkinsonian drugs, an increase in axial motor disturbances (postural instability, hardening, hypomimia, dystonia, dysarthria, bradykinesia, camptocormia, autonomic dysfunction, sleep disturbances) [7].

Cognitive impairment is one of the main factors that affects the quality of life of patients, dramatically aggravates and makes it impossible to care for the family; worsen the outlook and dramatically increase the risk of developing dopaminergic psychotic disorders in the background of therapy [11].

\section{Aim of the research}

To study the peculiarities of cognitive impairment in patients with PD and autoimmune thyroiditis (AIT), their association with motor and non-motor disorders, and to assess the impact on the quality of life of patients.

\section{Materials and methods of the research}

The research was conducted at the Department of Neurology No. 1 of the Shupyk National Medical Academy of Postgraduate Education in 2014-2018. A set of patients took place during a consultative review of the direction of the neurologists in the city of Kyiv and the Kyiv region, in the neurological department of the Kyiv City Clinical Hospital No. 9, in the neurological department No. 1 of the Kyiv City Clinical Hospital No. 8. A comprehensive clinical-paraclinical examination of 109 patients with PD aged 47 to 75 years (mean age $61.9 \pm 0.6$ ). The main group consisted of patients with IA and IB subgroups, control - IIA and IIB subgroups. The IA subgroup consisted of 22 patients (mean age 57.68 1.06 ) with PD and AIT who did not receive pathogenetic antiparkinsonian therapy; subgroup of IB - 37 patients (mean age $63.65 \pm 0.76$ ) with PD and AIT, which for a long period received pathogenetic antiparkinsonian therapy; subgroup IIA - 23 patients (mean age $58.17 \pm 1.35$ ) on PD who did not receive pathogenetic antiparkinsonian therapy; subgroup $\mathrm{IIb}$ - patients with prolonged duration of PD, who were on antiparkinsonian therapy, in a number of 27 people (mean age 66.11 \pm 1.02 ). The diagnosis of Parkinson's disease was determined in accordance with the clinical and diagnostic criteria of the Brain Bank of the British Society of Parkinson's Disease, according to which Parkinsonism can be diagnosed in the presence of akinesia (bradykinesia) and one of the symptoms: rigidity of muscles, resting tremor, or postural instability, which more often joins on more late stages of the disease, not related to the primary visual impression, vestibular, cerebellar, proprioceptive dysfunction.

General clinical and neurological examinations, evaluation of motor functions by the Unified Parkinson's disease Rating Scale PD (UPDRS), neuropsychological testing (MMSE, MoCA, FAB, BDI), Hamilton's Alert Scale (HARS), scale used for assessing autonomic disorders in patients with PD and Parkinson's disease questionnaire (PDQ-39).

In the statistical analysis, the program "Statistica 6.0" was used. Data processing and analysis was carried out in OpenOffice software packages (Base, Calc, Writer, Draw, Math), GNU 
Octave (with preservation of *.doc, *.xls files) - open source software, its use is regulated by the license GPL (General Public License) and IBM SPSS from cloud. The quantitative values were represented as median (Me), interquartile scope (IQR: Q1-Q3), also determined the scope (Range: min-max). When comparing the two groups, the Mann-Whitney (U) criterion was used for independent groups. For the analysis of the direction and the strength of the relationship between the quantitative indicators, the method of correlation analysis was used to calculate the Spirman correlation coefficient $(\rho)$.

\section{Results of the research}

Comparing the results of the neuropsychological testing of the IA subgroup with the results of the IIA subgroup in the primary examination revealed statistically significant lower rates in the subgroup IA: FAB (Me 16.0; IQR: 15.0-17.0; Range: 15.0-18.0; Me 17.0; IQR: 17.0-18.0; Range : 15.0-18.0; U=145.5; $\mathrm{p}=0.011$ ). MoCA (Me 25.5; IQR: 25.0-26.0; Range: 25.0-27.0 and Me 26.0; IQR: 26.0-27.0; Range: 25.0-30.0; $U=162.5 ; \mathrm{p}=0.028$ ). the clock drawing test (Me 7.5; IQR: 6.0-9.0; Range: 5.0-10.0; Me 9.0; IQR: 9.0-10.0; Range: 7.0-10.0; U=111.5; $\mathrm{p}=0.001$ ).

Analyzing the MMSE scale in the subgroup of IA and IIA in the primary examination (Me 27.0; IQR: 26.8-28.0; Range: 26.0-28.0; Me 27.0; IQR: 27.0-30.0; Range: 26.0-30.0; U=178.0; $\mathrm{p}=0.075$ ) and a year later (Me 27.0; IQR: 26.8-28.0; Range: 26.0-28.0 and Me 27.0; IQR: 27.030.0; Range: 26.0-30.0; U=178.0; $\mathrm{p}=0.075)$; no statistically significant difference was detected. Indicators of neuropsychological tests in patients with PD, depending on the presence of AIT in the primary review, are presented in Table 1

Table 1

Indicators of neuropsychological tests in patients with PD depending on the presence of AIT in the primary examination

\begin{tabular}{|c|c|c|c|c|c|c|c|c|}
\hline \multirow{4}{*}{ Scale } & \multicolumn{2}{|c|}{ IA subgroup } & \multicolumn{2}{|c|}{ IB subgroup } & \multicolumn{2}{|c|}{ IIA subgroup } & \multicolumn{2}{|c|}{ IIB subgroup } \\
\hline & Q1 & & Q1 & & Q1 & & Q1 & \\
\hline & Me & Min & Me & Min & Me & Min & Me & Min \\
\hline & Q3 & Max & Q3 & $\operatorname{Max}$ & Q3 & $\operatorname{Max}$ & Q3 & $\operatorname{Max}$ \\
\hline \multirow{3}{*}{ MMSE (points) } & 26.8 & & 25.0 & & 27.0 & & 26.0 & \\
\hline & 27.0 & 26.0 & 26.0 & 23.0 & 27.0 & 26.0 & 27.0 & 25.0 \\
\hline & 28.0 & 28.0 & 26.5 & 27.0 & 30.0 & 30.0 & 27.0 & 28.0 \\
\hline \multirow{3}{*}{ FAB (points) } & 15.0 & & 14.0 & & 17.0 & & 15.0 & \\
\hline & 16.0 & 15.0 & 15.0 & 14.0 & 17.0 & 15.0 & 16.0 & 14.0 \\
\hline & 17.0 & 18.0 & 15.0 & 17.0 & 18.0 & 18.0 & 17.0 & 18.0 \\
\hline \multirow{3}{*}{ MoCA (points) } & 25.0 & & 24.0 & & 26.0 & & 25.0 & \\
\hline & 25.5 & 25.0 & 25.0 & 22.0 & 26.0 & 25.0 & 25.0 & 24.0 \\
\hline & 26.0 & 27.0 & 25.5 & 27.0 & 27.0 & 30.0 & 26.0 & 27.0 \\
\hline \multirow{3}{*}{$\begin{array}{l}\text { The clock drawing } \\
\text { test (points) }\end{array}$} & 6.0 & & 7.0 & & 9.0 & & 7.0 & \\
\hline & 7.5 & 5.0 & 7.0 & 5.0 & 9.0 & 7.0 & 9.0 & 7.0 \\
\hline & 9.0 & 10.0 & 8.5 & 9.0 & 10.0 & 10.0 & 9.0 & 9.0 \\
\hline \multirow{3}{*}{$\begin{array}{l}\text { Phonetic speed of } \\
\text { speech (words) }\end{array}$} & 9.0 & & 7.0 & & 9.0 & & 9.00 & \\
\hline & 9.5 & 8.0 & 8.0 & 6.0 & 10.0 & 8.0 & 9.00 & 6.0 \\
\hline & 11.0 & 15.0 & 9.0 & 11.0 & 12.0 & 12.0 & 11.00 & 14.0 \\
\hline \multirow{3}{*}{$\begin{array}{l}\text { Postponed memory } \\
\text { (words) }\end{array}$} & 2.0 & & 2.0 & & 2.0 & & 2.0 & \\
\hline & 3.0 & 1.0 & 2.0 & 2.0 & 3.0 & 2.0 & 2.0 & 2.0 \\
\hline & 3.0 & 3.0 & 3.0 & 3.0 & 3.0 & 5.0 & 3.0 & 4.0 \\
\hline
\end{tabular}


After 1 year, the data of neuropsychological testing in patients with IA and IIA subgroups deteriorated, statistically significant lower values were stored in the subgroup IA: FAB (Me 16.0; IQR: 15.0-17.0; Range: 15.0-17.0; Me 17.0; IQR: 17.0-18.0; Range: 15.0-18.0; U=132.5; $\mathrm{p}=0.004)$. MoCA (Me 25.0; IQR: 25.0-26.3; Range: 24.0-27.0 and Me 26.0; IQR: 26.0-27.0; Range: 25.030.0; $\mathrm{U}=152.0 ; \mathrm{p}=0.017$ ); clock drawing test (Me 6.5; IQR: 5.8-9.0; Range: 5.0-10.0 and Me 9.0; IQR: 8.0-10.0; Range: 7.0-10.0; $\mathrm{U}=110.5 ; \mathrm{p}=0.001)$.

By analyzing the sub-tests of the MoCA scale (phonetic speed of speech, postponed memory), no statistically significant difference was found between the indices of the IA and IIA subgroups in the initial examination (phonetic speed of speech: Me 9.5; IQR: 9.0-11.0; Range: 8.0-15.0 and Me 10.0; IQR: 9.0-12.0; Range: 8.0-12.0; $\mathrm{U}=206.0 ; \mathrm{p}=0.27)$, postponed memory: Me 3.0; IQR: 2.0-3.0; Range: 1.0-3.0 and Me 3.0; IQR: 2.0-3.0; Range: 2.0-5.0; $U=226.0, \mathrm{p}=0.5$ ).

After 1 year, there was no statistically significant difference between the sub-tests of the MoCA scale in the subgroups of IA and IIA (phonetic speech rate: Me 8.5; IQR: 7.0-11.0; Range: 7.0-13.0 and Me 10.0; IQR: 10.0-11.0; Range: 7.0-11.0; U=170.0; p=0.06), postponed memory: Me 3.0; IQR: 2.0-3.0; Range: 2.0-3.0 and Me 3.0; IQR: 2.0-3.0; Range: 2.0-5.0; U=252.0, p=0.98).

The analysis of the results of the neuropsychological testing in the subgroups IB and IB in the initial survey showed statistically significant lower rates in the subgroup IB: MMSE (Me 26.0; IQR: 25.0-26.5; Range: 23.0-27.0 and Me 27.0; IQR: 26.0-27.0; Range: 25.0-28.0; U=260.5; $\mathrm{p}=0.001$ ). FAB (Me 15.0; IQR: 14.0-15.0; Range: 14.0-17.0 and Me 16.0; IQR: 15.0-17.0; Range: 14.0-18.0; U=276.0; $\mathrm{p}=0.002$ ). MoCA (Me 25.0; IQR: 24.0-25.5; Range: 22.0-27.0; Me 25.0; IQR: 25.0-26.0; Range: 24.0-27.0; $U=308.5 ; \mathrm{p}=0.007$ ), clock drawing test (Me 7.0; IQR: 7.0-8.5; Range: 5.0-9.0 and Me 9.0; IQR: 7.0-9.0; Range: 7.0-9.0; U=284.5; p=0.002).

After 1 year, statistically significant lower neuropsychological tests were observed in IB subgroup patients compared to the IIB subgroup: MMSE (Me 25.0; IQR: 24.0-26.0; Range: 23.027.0 and Me 26.0; IQR: 26.0-27.0; Range: 24.0-28.0; U=190.0, p=0.001). FAB (Me 14.0; IQR: 14.0-15.0; Range: 13.0-16.0 and Me 16.0; IQR: 15.0-16.0; Range: 14.0-17.0; $U=217.0 ; p=0.001)$. MoCA (Me 24.0; IQR: 23.0-25.0; Range: 22.0-26.0 and Me 25.0; IQR: 24.0-25.0; Range: 24.027.0; $\mathrm{U}=303.0 ; \mathrm{p}=0.005$ ), the clock drawing test (Me 7.0; IQR: 6.0-8.0; Range: 6.0-9.0 and Me 8.0; IQR: 7.0-8.0; Range: 6.0-9.0; $\mathrm{U}=308.5 ; \mathrm{p}=0.007$ ). Indicators of neuropsychological tests in patients with PD depending on the presence of AIT in a year are shown in Table 2.

\section{Table 2}

Indicators of neuropsychological tests in patients with PD depending on the presence of AIT in a year

\begin{tabular}{|c|c|c|c|c|c|c|c|c|}
\hline \multirow{4}{*}{ Scale } & \multicolumn{2}{|c|}{ IA subgroup } & \multicolumn{2}{|c|}{ IB subgroup } & \multicolumn{2}{|c|}{ IIA subgroup } & \multicolumn{2}{|c|}{ IIB subgroup } \\
\hline & Q1 & & Q1 & & Q1 & & Q1 & \\
\hline & Me & Min & Me & Min & Me & Min & Me & Min \\
\hline & Q3 & Max & Q3 & Max & Q3 & Max & Q3 & Max \\
\hline \multirow[t]{2}{*}{1} & 2 & 3 & 4 & 5 & 6 & 7 & 8 & 9 \\
\hline & 26.8 & & 24.0 & & 27.0 & & 26.0 & \\
\hline \multirow[t]{3}{*}{ MMSE (points) } & 27.0 & 26.0 & 25.0 & 23.0 & 27.0 & 26.0 & 26.0 & 24.0 \\
\hline & 28.0 & 28.0 & 26.0 & 27.0 & 30.0 & 30.0 & 27.0 & 28.0 \\
\hline & 15.0 & & 14.0 & & 17.0 & & 15.0 & \\
\hline \multirow[t]{3}{*}{ FAB (points) } & 16.0 & 15.0 & 14.0 & 13.0 & 17.0 & 15.0 & 16.0 & 14.0 \\
\hline & 17.0 & 17.0 & 15.0 & 16.0 & 18.0 & 18.0 & 16.0 & 17.0 \\
\hline & 25.0 & & 23.0 & & 26.0 & & 24.0 & \\
\hline \multirow[t]{2}{*}{ MoCA (points) } & 25.0 & 24.0 & 24.0 & 22.0 & 26.0 & 25.0 & 25.0 & 24.0 \\
\hline & 26.3 & 27.0 & 25.0 & 26.0 & 27.0 & 30.0 & 25.0 & 27.0 \\
\hline \multirow{3}{*}{$\begin{array}{l}\text { The clock drawing } \\
\text { test (points) }\end{array}$} & 5.8 & & 6.0 & & 8.0 & & 7.0 & \\
\hline & 6.5 & 5.0 & 7.0 & 6.0 & 9.0 & 7.0 & 8.0 & 6.0 \\
\hline & 9.0 & 10.0 & 8.0 & 9.0 & 10.0 & 10.0 & 8.0 & 9.0 \\
\hline
\end{tabular}




\begin{tabular}{ccccccccc}
\hline $\mathbf{1}$ & $\mathbf{2}$ & $\mathbf{3}$ & $\mathbf{4}$ & $\mathbf{5}$ & $\mathbf{6}$ & $\mathbf{7}$ & $\mathbf{8}$ & $\mathbf{9}$ \\
\hline \multirow{2}{*}{$\begin{array}{c}\text { Phonetic speed of } \\
\text { speech (words) }\end{array}$} & 7.0 & & 6.0 & & 10.0 & & 8.0 & 7.0 \\
& 11.5 & 7.0 & 7.0 & 5.0 & 10.0 & 7.0 & 8.0 & \\
& 2.0 & 13.0 & 8.0 & 9.0 & 11.0 & 11.0 & 10.0 & 11.0 \\
Postponed memory & 3.0 & 2.0 & 2.0 & 1.0 & 3.0 & 2.0 & 2.0 & 2.0 \\
(words) & 3.0 & 3.0 & 3.00 & 3.0 & 3.0 & 5.0 & 3.0 & 4.0
\end{tabular}

Analyzing the indicator of the MMSE scale in dynamics, a statistically significant difference was found in the subgroups of IB $(z=-3.95, p=0.001)$ and IIB $(z=-2.27, p=0.02)$, in subgroups of IA ( $\mathrm{z}=0.001, \mathrm{p}=1.0)$ and IIA $(\mathrm{z}=-0.001, \mathrm{p}=1.0)$ - was not observed (Fig. 1).

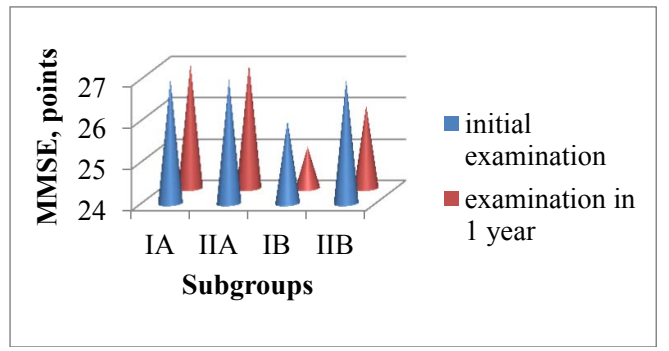

Fig. 1. The MMSE scale indicator in the dynamics subgroups

In the subgroups of the IB $(z=-3.56, p=0.001)$ and IIB $(z=-3.16, p=0.002)$, a statistically significant difference of the FAB indicator in the dynamics was revealed. In IA $(\mathrm{z}=-0.33, \mathrm{p}=0.74)$ and IIA ( $\mathrm{z}=0.001, \mathrm{p}=1.00)$ - it was not observed (Fig. 2).

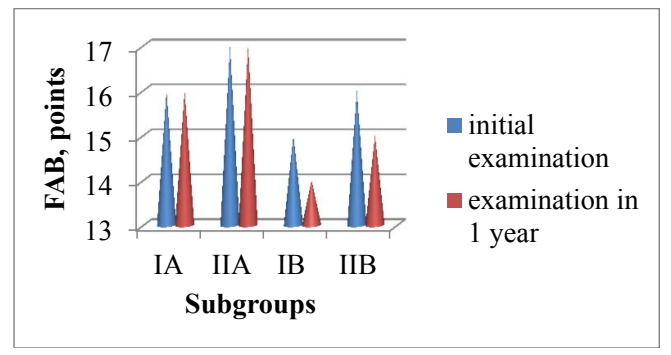

Fig. 2. The FAB scale indicator in the dynamics subgroups

Comparing the MoCA index with dynamics, a statistically significant difference was found in the subgroups of IB $(z=-3.95, p=0.001)$ and IIB $(z=-2.45, p=0.014)$. In subgroups of IA $(z=-1.13$, $\mathrm{p}=0.26)$ and IIA $(\mathrm{z}=0.001, \mathrm{p}=1.00)-$ not observed (Fig. 3).

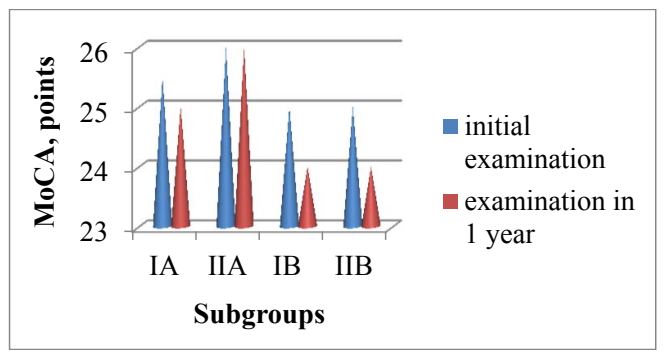

Fig. 3. The scale of the MoCA in the subgroups of the comparison in dynamics

The analysis of the clock drawing test in the dynamics showed a statistically significant difference in the subgroups of the IA $(\mathrm{z}=-2.45, \mathrm{p}=0.014)$, IB $(\mathrm{z}=-2.45, \mathrm{p}=0.014)$. In the sub- 
groups IIA $(\mathrm{z}=-1.41, \mathrm{p}=0.16)$ and IIB $(\mathrm{z}=-1.36, \mathrm{p}=0.15)$, no statistically significant difference was detected.

Comparing sub-tests of the MoCA scale (phonetic speed of speech, postponed memory), statistically significant lower values of the phonetic speed of the language of the IB subgroup were found compared to the IIB subgroup in the initial examination (Me 8.0; IQR: 7.0-9.0; Range: 6.0-11.0 and Me 9.0; IQR: 9.0-11.0; Range: 6.0-14.0; $\mathrm{U}=254.5 ; \mathrm{p}=0.001)$ and a year later (Me 7.0; IQR: 6.0-8.0; Range: 5.0-9.0; Me 8.0; IQR: 8.0-10.0; Range: 7.0-11.0; U=231.0; $\mathrm{p}=0.001$ ).

Comparing the MoCA, postponed memory of IB and IB subgroup metrics in the primary review (Me 2.0; IQR: 2.0-3.0; Range: 2.0-3.0 and Me 2.0; IQR: 2.0-3.0; Range: 2.0-4.0); and a year later (Me 2.0; IQR: 2.0-3.0; Range: 1.0-3.0 and Me 2.0; IQR: 2.0-3.0; Range: 2.0-4.0). No statistically significant difference was found $(U=466.0, p=0.59$ and $U=430.0, p=0.27$, respectively).

Analyzing the sub-tests of the scale of MoCA (phonetic speed of speech, postponed memory) in dynamics, certain features were revealed. There was a statistically significant difference in the indicators of the phonetic speed of the language in the subgroups of the IA $(z=-3.50, p=0.001)$, IB $(\mathrm{z}=-4.44, \mathrm{p}=0.001)$, IIB ( $\mathrm{z}=-3.37, \mathrm{p}=0.001)$; In the IIIA subgroup, no statistically significant difference was found $(\mathrm{z}=-1.50, \mathrm{p}=0.13)$. Analysis of the indicator of delayed memory in the dynamics showed a statistically significant difference in subgroups IA $(z=-2.00, p=0.046)$ and IB $(\mathrm{z}=-2.45, \mathrm{p}=0.014)$. In the subgroups IIA $(\mathrm{z}=0.001, \mathrm{p}=1.00)$ and IIB $(\mathrm{z}=-1.41, \mathrm{p}=0.16)$, no statistically significant difference was detected.

During the analysis of the results of the neuropsychological testing, there was no association at the statistically significant level in the subgroups of IA and IB between the duration of the disease and the MMSE scale $(\rho=0.32, p=0.06$ and $\rho=0.31, p=0.06$ respectively), the indicator of the FAB scale $(\rho=0.31, p=0.16$ and $\rho=0.009, p=0.96$, respectively), the indicator of the MoCA scale ( $\rho=0.34, p=0.12$ and $\rho=0.22, p=0.18$, respectively), an indicator of the phonetic speed of the language $(\rho=0.42, p=0.05$ and $\rho=0.13, p=0.44)$, the index of postponed memory $(\rho=0.21, p=0.45$ and $\rho=0.3, p=0.07$ ); there was a direct connection at a statistically significant level of average strength between the duration of the disease and the index of the clock drawing test $(\rho=0.51, p=0.03$ and $\rho=0.39, p=0.02$ ).

In the IA subgroups, a direct correlation was found between the statistically significant level of average strength between the age of the onset of the disease and the MMSE scale $(\rho=0.32$, $p=0.048)$, the index of the MoCA scale $(\rho=0.42, p=0.045)$, the indicator the scale of the FAB $(\rho=0.44, p=0.04)$, the index of the clock drawing test $(\rho=0.46, p=0.03)$, the index of phonetic speed of the speech $(\rho=0.51, p=0.02)$; There was no observed relationship at the statistically significant level between the age of the onset of the disease and the amount of delayed memory $(\rho=0.17$, $p=0.45$ ). In the IB subgroup, there was no statistically significant relationship between the age of the onset of the disease and the MMSE scale $(\rho=-0.26, p=0.25)$, the FAB $(\rho=0.21, p=0.22)$, indicator of the MoCA scale $(\rho=0.20, p=0.23)$; an indicator of the phonetic speed of speech $(\rho=0.20$, $\mathrm{p}=0.23)$, an indicator of postponed memory $(\rho=0.21, \mathrm{p}=0.21)$; A direct connection was found at a statistically significant level of average strength between the age of the onset of the disease and the clock drawing test $(\rho=0.44, p=0.007)$.

In the IA subgroup, during the primary examination and after a year, no link was found at the statistically significant level between the level of depression and the indicator of the MMSE scale $(\rho=0.20, p=0.36$ and $\rho=0.29, p=0.18$, respectively $)$, the index of the MoCA scale $(\rho=0.20$, $p=0.38$ and $\rho=0.13, p=0.56$, respectively), the index of the FAB scale $(\rho=0.03, p=0.90$ and $\rho=-0.10, p=0.67$, respectively). In the IA subgroup, in the initial review, feedback was observed at a statistically significant level of average strength between the level of anxiety and the MMSE scale $(\rho=-0.60, p=0.003)$, the index of the MoCA scale $(\rho=-0.46, p=0.03)$; between the Hamilton scale and the FAB scale was not detected $(\rho=0.04, p=0.88)$. A year later, in the IA subgroup, there was no observed correlation between the level of anxiety and the indicator of the MMSE scale $(\rho=0.35, p=0.18)$, the index of the MoCA scale $(\rho=0.13, p=0.56)$, an indicator of the FAB scale $(\rho=-0.46, p=0.11)$.

The analysis of the relationship between the general indicator of autonomic disturbances and the indicator of the MMSE scale $(\rho=-0.10, p=0.66)$, the indicator of the MoCA scale $(\rho=0.07$, 
$\mathrm{p}=0.75)$, the FAB $(\rho=-0.34, \mathrm{p}=0.12)$ did not reveal a statistically significant difference in the IA subgroup in the primary examination. A year later there was a feedback on a statistically significant level of average strength between the general indicator of vegetative disorders and the FAB scale $(\rho=-0.59, p=0.004)$; between the general index of vegetative scale and the MMSE scale $(\rho=-0,004$, $p=0.99)$, the indicator of the MoCA scale $(\rho=-0.12, p=0.59)$ of communication at the statistically significant level was not found.

In the IA subgroup, during the primary examination and after a year, no link was found at the statistically significant level between the overall score scale UPDRS and the MMSE scale ( $\rho=-0.06, p=0.80$ and $\rho=-0.08, p=0.72$, respectively), the indicator of the MoCA scale $(\rho=-0.26$, $p=0.25$ and $\rho=-0.24, p=0.29$, respectively), the index of the FAB scale $(\rho=-0.42, p=0.05$ and $\rho=0.21, p=0.34$, respectively).

In the IA subgroup, during the primary examination and after a year, a direct correlation was found between the statistically significant level of the strong force between the total quality of life of patients on PD (PDQ-39) and the MMSE scale ( $\rho=0.97, p=0.001$ and $\rho=0.90, p=0.001$, respectively), the indicator of the MoCA scale $(\rho=0.87, p=0.001$ and $\rho=0.85, p=0.001$, respectively); a direct connection at a statistically significant level of average strength between the total quality of life of patients on PD and the FAB scale $(\rho=0.45, p=0.03$ and $\rho=0.44, p=0.03$, respectively).

Statistically significant feedback of average strength was found in the IB subgroup at the primary examination and one year between the Beck scale score and the MMSE test result $(\rho=-0.97$, $p=0.001$ and $\rho=-0,90, p=0.001$, respectively), the indicator of the MoCA scale $(\rho=-0.56, p=0.001$ and $\rho=-0.54, p=0.001$, respectively), the index of the FAB scale $(\rho=-0.40, p=0.045$ and $\rho=-0.32$, $\mathrm{p}=0.045$, respectively).

Feedback was received at a statistically significant level of average strength in the IB subgroup in the primary examination and one year between the level of anxiety and the MMSE scale ( $\rho=-0.52, p=0.001$ and $\rho=-0.35, p=0,03$, respectively), the indicator of the MoCA scale $(\rho=-0.55$, $p=0.001$ and $\rho=-0.54, p=0.001$, respectively), the index of the FAB scale $(\rho=-0.40, p=0.045$ and $\rho=0.54, p=0.001$, respectively).

There was a feedback on a statistically significant level of average strength in the IB subgroup during primary examination and one year between the general indicator of autonomic disturbances and the MMSE scale indicator $(\rho=-0.65, p=0.001$ and $\rho=P=0.001$, respectively), the indicator of the MoCA scale ( $\rho=-0.66, p=0.001$ and $\rho=-0.64, p=0.001$, respectively), FAB scale index ( $\rho=-0.44, p=0.01$ and $\rho=-0.42, p=0.01$, respectively).

In the subgroup IB at the initial review and after a year, feedback was found on a statistically significant average power level between the total score scale of the UPDRS and the MMSE scale indicator $(\rho=-0.4, p=0.02$ and $\rho=-0.38, p=0.02$ respectively), the indicator of the MoCA scale ( $\rho=-0.38, p=0.02$ and $\rho=-0.64, p=0.001$, respectively), the indicator of the FAB $(\rho=-0.42, p=0.01$ and $\rho=-0.44, p=0.01$, respectively).

In the subgroup of IB during the initial review and after a year, feedback was found on a statistically significant average power level between the total patient quality of life (PDQ-39) and the MMSE scale $(\rho=-0.47, p=0.003$ and $\rho=-0.40, p=0.01$, respectively), the indicator of the MOS scale $(\rho=-0.34, p=0.04$ and $\rho=-0.36, p=0.03$, respectively). We did not notice a connection at a statistically significant level in the primary review and after a year between the total quality of life of patients on PD and the FAB score $(\rho=0.02, p=0.93$ and $\rho=-0.10, p=0.54$, respectively).

\section{Discussion of the results}

Studies show that in $80 \%$ of cases, dementia develops with a PD duration of more than 20 years. Dementia can be manifested in the form of dementia with Lewy bodies, neurodegeneration of dopaminergic and cholinergic cortical areas. Investigations of volumetric magnetic resonance imaging showed a posterior-cortical area atrophy in patients with moderate cognitive impairment and dementia [13]. Diffusion-tensor magnetic resonance imaging can detect changes in the white matter of the brain in the initial stages of cognitive deficits in patients with PD [14, 15]. Studies conducted on posthumous sections of the brain showed a significant loss of cholinergic neurons of subcortical nuclei, and surviving neurons contained inclusion, the body of Lewy. The 
activity of acetylcholinesterase, a presynaptic cholinergic marker, is significantly reduced in the frontal cortex in patients with dementia and without cognitive impairment in PD compared to the control group. There is a statistically significant relationship between the activity of cortical acetylcholinesterase and MMSE scale [16]. Another study showed cholinergic dysfunction of parietal and occipital areas in patients with PD, which did not show cognitive impairment [17]. In a study by Fengler S. and co-authors, it is shown: the reduction of the executive function can be considered an additional NMP of the prodromal phase of PD [18].

Our study showed moderate cognitive impairment and dementia in PD patients, depending on the duration of the disease. The lower rates of neuropsychological testing were observed in patients with PD and AIT. The high incidence of cognitive impairment in patients with PD is confirmed by previous clinical trials $[8,9]$.

We found a connection at a statistically significant level between the indicators of neuropsychological testing and NMS PD (anxiety, depression) in patients who received long-term antiparkinsonian therapy. A clinical study conducted by Nodel and co-authors shows a statistically significant relationship between the level of anxiety and the decrease in the control of cognitive functions, attention. There was also a link between the level of depression and the subjective evaluation of cognitive functions, especially in the early stages of the disease, while the link between self-esteem of cognitive impairment and objective changes (memory impairment) was statistically significant at deployed 3 stage PD. The pathophysiological basis of the relationship between cognitive and emotional-affective disorders may be different. Cognitive impairment and emotional-affective disturbances can be symptoms of a disease that develops in parallel with common pathophysiological mechanisms. Cognitive impairment can exacerbate or even cause anxiety and depression, and, conversely, emotional and affective disorders can negatively affect cognitive function [19].

Clinical significance of NMP is determined not only by the high frequency, but also by the degree of negative impact on the quality of life of patients. In one of the first studies of the impact of various PD symptoms on quality of life, a population-based Australian study showed that after 15 years of disease, the leading predictor of poor quality of life, besides depression, is dementia, which has a higher risk of extinction of psychosis [20]. Our study found a negative effect of the level of cognitive impairment on the quality of life of patients with PD and AIT, which is generally consistent with the results of a number of recent studies [21]. According to the results of a survey of patients with a recent diagnosis of PD, negative effects on quality of life, other than depression, anxiety, also carry out attention disorder and memory [22].

Such a significant effect of cognitive impairment on the quality of life of patients with PD causes the need for their diagnosis and appropriate correction.

\section{Conclusions}

1. Neuropsychological testing showed that the MMSE, MoCA, and FAB scores in patients with PD and AIT are lower in comparison with patients with PD.

2. There was a statistically significant difference in the dynamics of cognitive impairment in patients receiving long-term antiparkinsonian therapy.

3. There was a linkage between statistically significant levels of neuropsychological testing (MMSE, MoCA, FAB) and motor and non-motor manifestations of PD in patients receiving longterm antiparkinsonian therapy.

4. The negative influence of the level of cognitive impairment on the quality of life of patients with PD and AIT was revealed, indicating the high medical and social significance of these violations.

\section{References}

[1] Hanganu, A., Houde, J.-C., Fonov, V. S. Degroot, C., Mejia-Constain, B., Lafontaine, A.-L. et. al. (2018). White Matter Degeneration Profile in the Cognitive Cortico-Subcortical Tracts in Parkinson's Disease. Movement Disorders, 33 (7), 1139-1150. doi: http://doi.org/10.1002/mds.27364

[2] Berg, D., Postuma, R. B., Bloem, B., Chan, P., Dubois, B., Gasser, T. et al. (2014). Time to redefine PD? Introductory statement of the MDS Task Force on the definition of Parkinson's disease. Movement Disorders, 29 (4), 454-462. doi: http://doi.org/10.1002/mds.25844 
[3] Goldman, J. G., Postuma, R. (2014). Premotor and nonmotor features of Parkinson's disease. Current Opinion in Neurology, 27 (4), 434-441. doi: http://doi.org/10.1097/wco.0000000000000112

[4] Schrag, A., Horsfall, L., Walters, K., Noyce, A., Petersen, I. (2015). Prediagnostic presentations of Parkinson's disease in primary care: a case-control study. The Lancet Neurology, 14 (1), 57-64. doi: http:// doi.org/10.1016/s1474-4422(14)70287-x

[5] Barone, P., Antonini, A., Colosimo, C., Marconi, R., Morgante, L., Avarello, T. P. et. al. (2009). The PRIAMO study: A multicenter assessment of nonmotor symptoms and their impact on quality of life in Parkinson's disease. Movement Disorders, 24 (11), 1641-1649. doi: http://doi.org/10.1002/mds.22643

[6] Delgado-Alvarado, M., Gago, B., Navalpotro-Gomez, I., Jiménez-Urbieta, H., RodriguezOroz, M. C. (2015). Biomarkers for dementia and mild cognitive impairment in Parkinson's disease. Movement Disorders, 31 (6), 861-881. doi: http://doi.org/10.1002/mds.26662

[7] Levin, O. S., Fedorova, N. V. (2014). Parkinson's disease. Moscow: MEDpress-inform, 384.

[8] Aarsland, D. (2016). Cognitive impairment in Parkinson's disease and dementia with Lewy bodies. Parkinsonism \& Related Disorders, 22 (1), 144-148. doi: http://doi.org/10.1016/j.parkreldis.2015.09.034

[9] Arnaldi, D., De Carli, F., Famà, F., Brugnolo, A., Girtler, N., Picco, A. et al. (2017). Prediction of Cognitive Worsening in De Novo Parkinson's Disease Clinical Use of Biomarkers. Movement Disorders, 32 (12), 1738-1747. doi: http://doi.org/10.1002/mds.27190

[10] Anang, J. B. M., Gagnon, J.-F., Bertrand, J.-A., Romenets, S. R., Latreille, V., Panisset, M. et. al. (2014). Predictors of dementia in Parkinson disease: A prospective cohort study. Neurology, 83 (14), 1253-1260. doi: http://doi.org/10.1212/wnl.0000000000000842

[11] Aarsland, D., Andersen, K., Larsen, J. P., Lolk, A. (2003). Prevalence and Characteristics of Dementia in Parkinson Disease. Archives of Neurology, 60 (3), 387-392. doi: http://doi.org/10.1001/archneur.60.3.387

[12] Mak, E., Su, L., Williams, G. B., Firbank, M. J., Lawson, R. A., Yarnall, A. J. et. al. (2015). Baseline and longitudinal grey matter changes in newly diagnosed Parkinson's disease: ICICLE-PD study. Brain, 138 (10), 2974-2986. doi: http://doi.org/10.1093/brain/awv211

[13] Agosta, F., Canu, E., Stefanova, E., Sarro, L., Tomic, A., Spica, V. et. al. (2014). Mild cognitive impairment in Parkinson's disease is associated with a distributed pattern of brain white matter damage. Human Brain Mapping, 35 (5), 1921-1929. doi: http://doi.org/10.1002/hbm.22302

[14] Zheng, Z., Shemmassian, S., Shemmassian, S., Kim, W., Bookheimer, S. Y., Pouratian, N. (2014). DTI correlates of distinct cognitive impairmentsin Parkinson's disease. Human Brain Mapping, 35 (4), 1325-1333. doi: http://doi.org/10.1002/hbm.22256

[15] Ray Chaudhuri, K., Poewe, W., Brooks, D. (2018). Motor and Nonmotor Complications of Levodopa: Phenomenology, Risk Factors, and Imaging Features. Movement Disorders, 33 (6), 909-919. doi: http://doi.org/10.1002/mds.27386

[16] Baggio, H. C., Segura, B., Sala-Llonch, R., Marti, M.-J., Valldeoriola, F., Compta, Y. et. al. (2015). Cognitive impairment and resting-state network connectivity in Parkinson's disease. Human Brain Mapping, 36 (1), 199-212. doi: http://doi.org/10.1002/hbm.22622

[17] Fengler, S., Liepelt-Scarfone, I., Brockmann, K., Schäffer, E., Berg, D., Kalbe, E. (2017). Cognitive Changes in Prodromal Parkinson's Disease: A Review. Movement Disorders, 32 (12), 1655-1666. doi: http://doi.org/10.1002/mds.27135

[18] Nodel', M. R., Danilova, N. N., Glozman, Zh. M., Yakhno, N. N. (2016). The correlation of cognitive, emotional and affective disorders in patients with parkinson's disease. The Neurological Journal, 21 (6), 338-343. doi: http://doi.org/10.18821/1560-9545-2016-21-6-338-343

[19] Hely, M. A., Morris, J. G., Reid, W. G., Trafficante, R. (2005). Sydney multicenter study of Parkinson's Disease: non-L-dopa responsive problems dominate at 15 years. Movement Disorders, 20 (2), 190-199. doi: http://doi.org/10.1002/mds.20324

[20] Valkovic, P., Harsany, J., Hanakova, M., Martinkova, J., Benetin, J. (2014). Nonmotor Symptoms in Early- and Advanced-Stage Parkinson's Disease Patients on Dopaminergic Therapy: How Do They Correlate with Quality of Life? ISRN Neurology, 2014, 1-4. doi: http://doi.org/10.1155/2014/587302

[21] Duncan, G. W., Khoo, T. K., Yarnall, A. J., O’Brien, J. T., Coleman, S. Y., Brooks, D. J. et. al. (2014). Health-related quality of life in early Parkinson's disease: the impact of nonmotor symptoms. Movement Disorders, 29 (2), 195-202. doi: http://doi.org/10.1002/mds.25664 